\title{
Enacting evidence-based practice: pathways for community nurses.
}

\author{
TEODOROWSKI, P., CABLE, C., KILBURN, S., KENNEDY, C.
}

2019

This document is the Accepted Manuscript version of a Published Work that appeared in final form in British journal of community nursing, copyright $\odot$ MA Healthcare, after peer review and technical editing by the publisher. To access the final edited and published work see $h$ ttps://doi.org/10.12968/bjcn.2019.24.8.370. 


\section{Abstract}

Background: Community nurses are expected to deliver evidence based practice which is challenging given the diversity and breadth of the evidence base from which they can draw.

Aims: To explore community nurses' experiences of implementing change in their practice.

Methods: Qualitative semi-structured interviews $(n=9)$ and focus groups $(n=2)$ with community nurses $(n=17)$ were conducted.

Findings: Three pathways to introduce change in practice were identified by participants: bottom-up, top-down and collaborative pathways. These are based on the nature of the proposed change, the available evidence, 'buy in' from colleagues and issues around implementation.

Implications for practice and conclusion: These findings identify approaches to implementing change in community nursing practice. Practitioners would benefit from support to navigate the complex process of change through managerial support, ongoing education, accessible online resources and practice development support.

Keywords: Community nursing, evidence-based practice, EBP, evidence, research.

\section{Background}

Community nurses are a large and diverse group of the nursing workforce and their role includes preventive, therapeutic, supportive and end of life care. The evidence base they draw from to inform their practice includes a broad range of academic disciplines and knowledge. A commitment to ensuring that practice is evidence based is now firmly established whilst the challenges of implementing this in reality are well recognised and documented (Mathieson et al. 2018; Orton et al. 2011; NMC 2015; Thompson et al. 2005).

Evidence-based practice (EBP) evolved from evidence-based medicine and is defined by (Sackett et al. 1996:71) as 'the conscientious, explicit, and judicious use of current best evidence in making decisions about the care of individual patients'. Rycroft-Malone et al. 
(2004) go on to define evidence as a combination of research, clinical experience, patient experience and information from the local context. EBP demands that clinicians assume a questioning approach to practice and have skills in the analysis and synthesis of all forms of evidence. Clinicians then 'match' this to the individual needs, preferences and values of patients using their professional expertise (Greenhalgh et al. 2014). This is at the heart of the 'realistic' approach to care advocated by NHS England and Scotland's Chief Medical Officer, ensuring that the values and preferences of patients are central to care delivery (NHS England 2013; Scottish Government 2018).

Community nurses work with people, patients, families and communities so the transactional nature of the role and the need to respect individual preferences and choices adds to the complexity of ensuring practice is based on evidence (Pereira et al. 2018). It is therefore important that community nurses can access information to support their practice and can discern the utility of that evidence to inform their interactions with people, patients, families and communities.

This paper reports findings from a qualitative study conducted in Scotland with community nurses. The overall aim was to explore community nurse participants' experiences of EBP. The specific questions were to 1) explore participants' experiences of making a change in their practice, 2) establish what resources or information they required to achieve change, 3) identify what are 'hot topics' (e.g. variation in practice, evidence gap in their area of work) and 4) establish what assistance they may require to implement recent evidence and experience into practice.

\section{Methods}

This qualitative study utilised semi-structured interviews $(n=9)$ and focus groups $(n=2) ; 17$ community nurses took part in the study. The indicative topic guide was piloted and developed from feedback gathered using world café methods with practitioners and academics at the Queen's Nursing Institute Scotland Annual Conference in 2018. The questions aimed to 
explore participants' experiences of making a change in their practice; establish what resources or information they drew on, what assistance they may require, and identify current 'hot topics' (e.g. variation in practice, evidence gap in their area of work). All interviews and focus groups were recorded and transcribed. Data were analysed using framework analysis (Gale et al. 2013). The coding frame was developed inductively from the interviews, applied, and refined across all data. Codes exploring barriers and enablers on how to introduce EBP, accessing recent evidence and what can be done to support community nurses informed the identification of three themes (pathways). These emerged from the nature of the proposed change, the available evidence, 'buy in' from colleagues and issues around implementation.

\section{Participants}

Participants were community nurses based in one Health Board and/or associated with a School of Nursing and Midwifery in Scotland. Participants were drawn from a variety of specialisations including district nursing $(n=6)$, health visiting $(n=2)$, community mental health nursing $(n=1)$, community midwifery $(n=1)$, occupational health nursing $(n=2)$ and care home nursing $(n=5)$. Participants worked in both the private and public sectors and the participants included team leaders $(n=7)$. The approach to sampling was purposive and participants received an invitation letter to contact the researcher if they wished to participate.

\section{Limitations}

This study was relatively small and conduced within a Scottish context, which could limit the transferability of these findings. Community nurses are not one homogenous group and participants represented a variety, but not necessarily all community nursing roles.

\section{Ethics statement}

The University Research Ethics Committee approved this study. Oral or written consent was obtained from all participants. 


\section{Findings}

Three pathways to introduce change in practice were identified by participants: bottom-up, top-down and collaborative pathways. These are based on the nature of the proposed change, the available evidence, 'buy in' from colleagues and issues around implementation. These pathways are not mutually exclusive and one service change/improvement may utilise more than one pathway. Participants recognised that there are many evidence-based resources available. However, the volume of evidence available alongside time constraints meant evidence was not always used to shape services. This could result in community nurses being reactive rather than proactive in determining and enacting changes to their practice.

"It actually gets very difficult when you're on that treadmill, to be able to identify what would be better to make it easier and what changes are, are needed." (Health Visitor 1).

\section{Bottom-up pathway}

Participants reported that they initiated some practice changes through reading academic papers, conducting audits, responding to service user feedback, following training and through information on social media. Within this pathway, small but important changes were introduced. For example,

"I've implemented that community nurses have access to the orange waste bags, which seems really insignificant but, it was a big problem" (Mental Health Nurse)

These changes required managerial support. Participants felt the need to build their case on evidence relevant to the proposed change, the UK context and those that are cost neutral or may result in savings. This process was time consuming and participants recognised that they needed to bring others on board to implement and sustain changes.

You can suggest something, yes, and it might take a wee while before that changes but, you can't change practice yourself' (Community Midwife). 
Responding to feedback from patients, service users, clients or their families gathered through surveys, complaints or through expressions of gratitude was reported but the imitations were recognised.

"[patients] tend to provide, like, maybe letters or cards and how they felt during the time we're looking after them but, it's not a questionnaire, it's not a named review, nothing like that. It's that kind of feedback." (District Nurse 1)

Participants recognised that collecting feedback from older people, those with dementia or at the end of life was challenging and may be positive due to patients' concerns around ongoing care should they provide negative responses. Some participants felt senior management could control patient surveys, which they consider, could shape the content and limit opportunities to engage. Participants noted that collecting information from patient feedback that did not shape services was problematic.

"I don't know what actually happens to the data when it goes in (...) we have changed the data we collect because we felt we were collecting far too much data and there was never anything happening as a result so, we, the team leaders and manager collectively decided that we would reduce the data because health visitors were spending far too long sitting putting, you know, counting up all these numbers for it to, to disappear into the ether." (Health Visitor 1)

Local patient groups could identify issues or challenges and the following example demonstrates how nurses worked with them to find a workable solution.

"They wanted a GP practice but, it's quite a rural village and it's quite small(...)it wasn't possible to have a GP out there. So, the community formed a steering group and, along with the Scottish Government, I think they got involved at that point, just to see what, what they could do, and that's when I was approached, when I was a district nurse up there, to ask if we could do some nurse-led clinics up there (...) we had the facilities already. They had this little three-roomed consulting area, which was never used." (District Nurse 2). 
Participants reported using social media to stay up to date with recent evidence. Facebook was the most popular platform, as well as Twitter. There are a number of closed Facebook groups for community nurses, specific to one speciality e.g. district nursing, occupational health nursing or to a clinical issue e.g. infection control, infant feeding. Facebook was seen as a good opportunity to share information, experiences or ask a question of colleagues.

"it's (Facebook) really active as well so you can put your view point across and other people can put points across as well" (Occupational Health Nurse 2).

Participants recognised this evidence may not be peer-reviewed and lacks references so has limitations.

"it's anecdotal evidence so how good is it? Just 'cause someone says to do it, it's not evidence-based" (Health Visitor 2).

Participants pointed out that it was extremely difficult to set up any Facebook group in the NHS due to the paperwork required.

"Setting up anything is, is a logistical nightmare (...) If you want to set up a Facebook page you've to fill out something like a 30-page document to, erm, to justify what you're doing" (District Nurses Focus Group).

Volunteers run these groups in their own time and nurses may use their own phones or computers to participate. Access to a smartphone is also a barrier to using the many applications to support EBP available for mobile and tablet devices. Nurses spoke about a lack of confidentiality on social media and the need to ensure privacy around patient specific issues. Moderators would ask if a new member was a registered nurse, but they have no opportunity to check this. Twitter allowed nurses to follow organisations and specialists relevant to their clinical practice.

"The Faculty of Occupational Medicine, they tend to put new research papers or research evidence into medical guidelines that we follow (...) there might be changes in medical 
guidelines for sickness to do specific roles (...) I find that Twitter's the best place to actually find these, you know, find the links and it's very fast and up to date." (Occupational Health Nurse 1)

Participants spoke about attending training or local fora as a place to hear new ideas. However even when the training was free, nurses may not have be released to attend because of staff shortages. They suggested the need to develop interactive, online and practical training opportunities, and a practice development role.

Some nurses had to study in their free time. Thus, some participants proposed the need for a study day.

"Nurses should have a free study day that, you know, it doesn't mean that they won't be paid for it, they come to (the) home and study, you know (...) at the moment, we're doing our own studies in our own time" (Care Home Nurses)

The majority of participants read professional journals and some had personal subscriptions. Paper and online copies were equally popular. Online versions were generally accessed through a university (when a participant was also a postgraduate student) or public libraries. However, only one participant mentioned the Knowledge Network which is available to all NHS Scotland staff. Organisational websites, for example the Care Inspectorate, were a rich source of information for care home nurses.

Participants reported various ways of identifying new evidence. This included using a mix of sources depending on their personal preferences and the time and resources available. Taking the initiative to change practice is something participants in this study reported doing and they gleaned information from various sources to underpin this. There was a recognition that some of the information accessed was anecdotal rather than evidence based.

\section{Top-down-pathway}

Legislation and policy guidance has implications for nursing practice. Many participants saw this as "it was done to us and not with us" (Health Visitor 1) and others found some legislation 
and policy guidance to be out-of-date and prescriptive. Occupational health and health visiting were areas where legislation was described as influential.

"There was a change in blood pressure for group two driving so, medical guidelines changed the blood pressure readings so, again, we had to change our policies, you know, this is the level of blood pressure now, it wasn't what it was before, because it's a government guideline so, it can be as simple" (Occupational Health Nurse 1)

Keeping up to date with new guidance and policies was done through similar ways as the bottom-up pathway. Participants may have prioritised internal messages over other sources of information.

Management, in response to incidents, introduced some changes. One example was that of new requirements to support lone working. This had elicited both positive and negative responses amongst team members. Similarly, the introduction of a new referral system in one NHS area brought mixed responses with some participants questioning the basis of the changes. Sometimes changes introduced by senior management were viewed as poorly justified or as "cost saving" (District Nurses Focus Group).

The 'top down' pathway saw community nurses responding to directions to change although in many cases there remained scope to manage the implementation and evaluation at a local level.

\section{Collaborative pathway}

Key to sustaining change was working together in collaboration and as a team and this was the same for bottom up and top down changes.

"It's not just I decide to change something and then everybody else gets onboard, the three of us actually discuss it before we implement any changes" (Occupational Health Nurse 1) 
Raising ideas for change may happen during a team meeting and unless consensus can be achieved, then the change may not last.

"it's incredibly challenging. It's not so much the actual change, it's more the buy in the longevity of it so, people may do it for a short time and then they'll go back to their old ways again" (District Nurse 2).

Participants viewed the collaborative pathway as an efficient way to deal with time constraints by allowing individual staff members opportunities to share experiences. One example given was a journal club.

"four times a year we will have a journal club. So, what we'll do is we'll present, three of us will present something that we have found, or that we have looked at, an article or a piece of research, or something interesting that informs our practice and we'll bring that, and we'll have a discussion around it" (Health Visitor 1).

Sustaining these groups also brought challenges, particularly with staff shortages. Some suggested that they relied on students to keep them going.

"tends to be the trainees that do it because we are so short staffed, it's a vicious circle" (Health Visitor 2).

Participants suggested that moving from a top-down to a collaborative pathway may be possible through using a practice development approach, allowing team members to prioritise and agree local aims. An example was given of feedback through an anonymous staff questionnaire which enabled the team to reach consensus on the way forward which helped them to feel empowered and not just consulted. 


\section{Discussion}

The pathways presented in this study offer a way of thinking about approaches to identifying and implementing change in practice. These pathways are not hierarchical or mutually exclusive and elements overlap. An advantage of conceptualising approaches to change in this way, as opposed to the identification of facilitators and barriers to EBP, is the recognition that the nature of the change and the drivers for it differ. The evidence accessed by participants in this study was variable in terms of its potential rigour and trustworthiness. Participants in this study perceived lack of time as a major obstacle to identifying new evidence (Thompson et al. 2005). The challenges of heavy workloads and increasing expectations means participants required easily accessible, high-quality information although they recognised this may be anecdotal and thus not necessarily of high quality. This time pressure is all the more significant in light of the findings from a systematic review that calls for reflection as a core component of EBP (Mantzoukas 2008). Reflection and initiating a cycle of change requires protected time. Where the change is 'top down' this is likely to be underpinned by policy or legislation but still requires team working, reflection and consideration of how best to introduce and sustain the change.

There exists potential tensions between changes imposed through policy and legislation and changes practitioners initiate in response to service user feedback and community initiatives. Evidence of direct dialogue with people, patients, families and communities to identify 'bottom up' priorities did not emerge strongly in this study. In the case of bottom-up, and collaborative pathways, these were in the main nurse/manager driven. Nurses identified the need for change, built a case, obtained managerial support and delivered on a proposal. 
Findings from this study show the need to support community nurses to implement EBP. Sufficient time, organisational support and further education are key facilitators of EBP (Mallion and Brooke 2016). Resources need to be maximised to empower nurses to engage with best evidence both at clinical decision-making level in partnership with patients and in the local implementation of system wide changes. Digital access to materials and the support of nursing leaders emerged as important to participants in this study. Practical advice around challenges and enablers, and signposting to appropriate resources was viewed as lacking and support for nurses, especially those who had not recently undertaken formal study, was viewed as essential (Sadoughi et al. 2017). Participants in this study saw the creation of a practice development role, which could assist with evidence-based practice and improvement, as important.

Our findings suggest new resources should be accessible, available online and be concise. E-learning courses have been growing in popularity among nurses (Atack 2003) and recently online club journals started to emerge (Ferguson et al. 2017; Roberts et al. 2015). Videos summarising research or demonstrating practical skills were highlighted as a helpful resource. The need for interactive materials alongside access to a facilitator to address questions were other suggestions. Online materials should also be available as open access to enhance opportunities for reflection and thinking time. Participants identified that facilitators should understand and have experienced the realities of clinical practice. Participants reported they were often unaware of practice innovations being led by others or key topics relevant to them. Sharing more information between and across various groups of community nurses and other health professionals will help to use resources to best effect.

Participants were unable to access readily digital resources in work and they reported having to use personal phones or computers in their own time. Where participants had access to NHS IT they found materials blocked because of restrictions. 
Participants identified more funding was needed to support facilitated online groups such as Facebook for community nurses. Several organisations e.g. the Royal College of Nursing, We Communities and NHS Education for Scotland are already supporting groups that offer a safe discussion forum for community nurses. In addition, there are interprofessional fora for example the Primary Care Communities of Practice (Kislov et al. 2012). There is scope to further develop social media to continue to build clinical academic communities around evidence based practice.

\section{Implications for practice}

The findings from this study illuminate some of the issues surrounding the complex process of changing practice. Although we are suggesting three pathways, it is important to recognise that these are not mutually exclusive and there exists considerable overlap between these. Our findings demonstrate that community nurses are involved in the change process and experience some challenges in synthesising the range of evidence to make the case for change.

Findings also suggest that practitioners would benefit from support to navigate the complex process of change. Participants in this study identified their preferences for support through access to a facilitator to help them and through the provision of accessible online resources. There was recognition that change required team working and this was critical to both bottom up and top down initiatives.

\section{Conclusion}

Navigating change in practice is complex and practitioners understand the importance of this. Change required through the introduction of new legislation, guidance or policy requires a team approach to implementation. Where change is responsive to service user feedback or practitioner expert opinion, securing support from managers was viewed as essential if challenging. Regardless of the drivers for change, easy access to support was recognised as 
essential. The challenges of working across complex systems were identified and clear understanding of roles, a shared vision and service user involvement are necessary components of the change process alongside judicious use of best available evidence.

\section{Key points}

- Community nurses are expected to draw on evidence to shape their practice but face numerous obstacles, especially time constraints.

- The three pathways, bottom-up, top-down and collaborative pathways, can help to identify and implement evidence-based practice.

- The three pathways are not hierarchical or mutually exclusive and elements overlap.

- Practitioners would benefit from support to navigate through the process of change with a practice development role in community nursing and accessible online resources.

\section{CPD Reflective Questions}

1. Are there any online support tools such as Facebook group or Twitter account for community nurses in your geographic area or specialist area of practice?

2. Can you think of a recent change in practice you have been involved in and how the three pathways help to explain why and how this change came about?

3. Thinking about a recent change in practice can you identify the issues driving the need for change and those which may have hindered the change process?

4. What strategies do you use to keep up to date with the best available evidence?

\section{References}

Atack L. Becoming a web-based learner: Registered nurses' experiences. Journal of Advanced Nursing. 2003. 44(3):289-297. 
Ferguson C, DiGiacomo M, Gholizadeh L, Ferguson LE, Hickman LD. The integration and evaluation of a social-media facilitated journal club to enhance the student learning experience of evidence-based practice: A case study. Nurse Education Today. 2017. 48:123-128.

Gale NK, Heath G, Cameron E, Rashid S, Redwood S. Using the framework method for the analysis of qualitative data in multi-disciplinary health research. BMC Medical Research Methodology. 2013. 13(1):117.

Greenhalgh T, Howick J, Maskrey N. Evidence based medicine: A movement in crisis? BMJ : British Medical Journal. 2014. 348:g3725.

Kislov R, Walshe K, Harvey G. Managing boundaries in primary care service improvement: A developmental approach to communities of practice. Implement Sci. 2012. 7:97-97.

Mallion J, Brooke J. Community- and hospital-based nurses' implementation of evidence-based practice: Are there any differences? British Journal of Community Nursing. 2016. 21(3):148154.

Mantzoukas S. A review of evidence-based practice, nursing research and reflection: Levelling the hierarchy. Journal of Clinical Nursing. 2008. 17(2):214-223.

Mathieson A, Grande G, Luker K. Strategies, facilitators and barriers to implementation of evidencebased practice in community nursing: a systematic mixed-studies review and qualitative synthesis. Primary Health Care Research \& Development. 2018. 20(e6): 1-11.

NHS England. Putting patients first: The summary nhs england business plan for 2013/14 - 2015/16. 2013.

Nursing \& Midwifery Council. The Code. Professional standards of practice and behaviour for nurses, midwives and nursing associates. 2015.

Orton L, Lloyd-Williams F, Taylor-Robinson D, O'Flaherty M, Capewell S. The use of research evidence in public health decision making processes: Systematic review. 2011. 6(7):e21704-e21704. 
Pereira F, Pellaux V, Verloo H. Beliefs and implementation of evidence-based practice among community health nurses: A cross-sectional descriptive study. J Clin Nurs. 2018. 27:20522061

Roberts MJ, Perera M, Lawrentschuk N, Romanic D, Papa N, Bolton D. Globalization of continuing professional development by journal clubs via microblogging: A systematic review. J Med Internet Res. 2015. 17(4):e103.

Rycroft-Malone J, Seers K, Titchen A, Harvey G, Kitson A, McCormack B. What counts as evidence in evidence-based practice? Journal of Advanced Nursing. 2004. 47(1):81-90.

Sackett DL, Rosenberg WMC, Gray JAM, Haynes RB, Richardson WS. Evidence based medicine: What it is and what it isn't. BMJ. 1996. 312(7023):71.

Sadoughi F, Azadi T, Azadi T. Barriers to using electronic evidence based literature in nursing practice: A systematised review. Health Information \& Libraries Journal. 2017. 34(3):187199.

Scottish Government. Practising realistic medicine: Chief medical officer for scotland annual report. 2018.

Thompson C, McCaughan D, Cullum N, Sheldon T, Raynor P. Barriers to evidence-based practice in primary care nursing - why viewing decision-making as context is helpful. Journal of Advanced Nursing. 2005. 52(4):432-444. 